TRANSACTIONS OF THE

AMERICAN MATHEMATICAL SOCIETY

Volume 354, Number 7 , Pages 2797-2818

S 0002-9947(02)02895-7

Article electronically published on February 12, 2002

\title{
CHARACTERIZATION OF THE HILBERT BALL BY ITS AUTOMORPHISM GROUP
}

\author{
KANG-TAE KIM AND STEVEN G. KRANTZ
}

\begin{abstract}
Let $\Omega$ be a bounded, convex domain in a separable Hilbert space. The authors prove a version of the theorem of Bun Wong, which asserts that if such a domain admits an automorphism orbit accumulating at a strongly pseudoconvex boundary point, then it is biholomorphic to the ball. Key ingredients in the proof are a new localization argument using holomorphic peaking functions and the use of new "normal families" arguments in the construction of the limit biholomorphism.
\end{abstract}

\section{INTRODUCTION}

Bun Wong WON proved in 1977 that a strongly pseudoconvex domain in $\mathbb{C}^{n}$ with non-compact automorphism group must be the ball. In the intervening 22 years, there has been considerable work on this circle of ideas. Rosay [ROS] showed that (in order to draw the same conclusion) the domain can be allowed to be bounded, but arbitrary, and one need only assume that some boundary orbit accumulation point of the automorphism group action be strongly pseudoconvex.

Greene and Krantz [GK1] explored what happens when the orbit accumulation point is weakly pseudoconvex. Although the ideas developed in that paper have now been superseded by more modern methods that center around the technique of scaling (see [IK1, Ki4], KIKR] for a discursive treatment of the scaling method), certainly that paper ushered in a new avenue of inquiry. Subsequent work by Bedford/Pinchuk [BP1]-[BP4], by Frankel [Fr], by Kim [Ki2]-[Ki6], by $\mathrm{Fu} /$ Isaev/Krantz ([K2]-[IK4, [FIK1]-[FIK2]), by Berteloot and Coeuré ([Ber1]Ber3, $\mathrm{BeCo}$ ), and by many others has fleshed out this circle of ideas.

It should be stressed, however, that all work to date has been in complex spaces of finite dimensions. The most complete results are available in $\mathbb{C}^{2}$. The work in dimensions 3 and higher continues to develop. Nevertheless, some questions remain open in all dimensions 2 and higher.

In the present work we begin to explore a domain in an infinite-dimensional complex Hilbert space; we assume that the domain possesses an automorphism group orbit accumulating at a boundary point. [Notice that the mere non-compactness of the automorphism group with respect to the compact-open topology does not give the (rigid) structure that we require.] In particular, we shall formulate and prove a version of the Wong/Rosay theorem in that setting.

We are pleased to thank Yun-Sung Choi, Jaesung Lee, John McCarthy, and Richard Rochberg for helpful discussions. The work of Laszlo Lempert sparked our

Received by the editors January 20, 2000 and, in revised form, March 23, 2001.

2000 Mathematics Subject Classification. Primary 32A07.

(C)2002 American Mathematical Society 
interest in the holomorphic function theory of infinitely many variables, and his helpful advice is also much appreciated.

The first author's research was funded partly by Grant 981-0104-018-2 and Interdisciplinary Research Program Grant 1999-2-102-003-5 of the Korean Science and Engineering Foundation. The second author was supported in part by NSF Grants DMS-9531967 and DMS-9631359.

\section{Preliminaries}

Let $\mathcal{H}$ be an infinite-dimensional, separable Hilbert space over the complex numbers.

2.1. Derivatives and $C^{k}$ smoothness. First we consider the differentiability of functions. Let $W \subseteq \mathcal{H}$ be an open subset. Consider $u: W \rightarrow \mathcal{E}$, where $\mathcal{E}$ is some complex Hilbert space. For $\mathbf{q} \in W$ and $\mathbf{v}_{1}, \ldots, \mathbf{v}_{k} \in \mathcal{H}$, we consider the following derivatives:

$$
\begin{aligned}
& d u\left(\mathbf{q} ; \mathbf{v}_{1}\right)=\lim _{\mathbb{R} \ni \epsilon \rightarrow 0} \frac{1}{\epsilon}\left(u\left(\mathbf{q}+\epsilon \mathbf{v}_{1}\right)-u(\mathbf{q})\right), \\
& d^{2}\left(\mathbf{q} ; \mathbf{v}_{1}, \mathbf{v}_{2}\right)=\lim _{\mathbb{R} \ni \epsilon \rightarrow 0} \frac{1}{\epsilon}\left(d u\left(\mathbf{q}+\epsilon \mathbf{v}_{2} ; \mathbf{v}_{1}\right)-d u\left(\mathbf{q} ; \mathbf{v}_{1}\right)\right) \\
& \vdots \\
& d^{k}\left(\mathbf{q} ; \mathbf{v}_{1}, \ldots, \mathbf{v}_{k}\right)= \\
& \lim _{\mathbb{R} \ni \epsilon \rightarrow 0} \frac{1}{\epsilon}\left(d^{k-1} u\left(\mathbf{q}+\epsilon \mathbf{v}_{k} ; \mathbf{v}_{1}, \ldots, \mathbf{v}_{k-1}\right)-d^{k-1} u\left(\mathbf{q} ; \mathbf{v}_{1}, \ldots, \mathbf{v}_{k-1}\right)\right) .
\end{aligned}
$$

We say that the mapping $u$ is $C^{k}$ smooth (for $k \geq 1$ ) if each $d^{\ell} u, \ell=1, \ldots, k$, exists and is a continuous map of $W \times \underbrace{\mathcal{H} \times \ldots \times \mathcal{H}}_{\ell \text { copies }}$ into $\mathcal{E}$.

In this case, it is known that each $d^{\ell} u$ is a symmetric $\ell$-linear map over $\mathbb{R}$.

We may also consider the following derivatives:

$$
\begin{aligned}
& D u(\mathbf{q} ; \mathbf{v})=\frac{d u(\mathbf{q} ; \mathbf{v})-i d u(\mathbf{q} ; i \mathbf{v})}{2}, \\
& \bar{D} u(\mathbf{q} ; \mathbf{v})=\frac{d u(\mathbf{q} ; \mathbf{v})+i d u(\mathbf{q} ; i \mathbf{v})}{2} .
\end{aligned}
$$

Since a $C^{1}$ smooth function that is holomorphic in every complex direction is holomorphic (this is the Gateaux definition of holomorphicity, for which see $[\mathrm{Mu}]$ ), we see that every $C^{1}$ smooth mapping $u$ with $\bar{D} u$ identically zero is holomorphic. An alternative, and equivalent, definition is to demand that $u$ be holomorphic on each finite-dimensional slice. Both of these are equivalent to specifying that $u$ have a power series expansion about each point.

2.2. Strong pseudoconvexity. By a domain in $\mathcal{H}$, we mean a connected open subset of $\mathcal{H}$. If $\Omega$ is a domain in $\mathcal{H}$, then we denote its boundary by $\partial \Omega$. We say that a boundary point $\mathbf{p} \in \partial \Omega$ is $C^{k}$ smooth $(k \geq 1)$ if $\mathbf{p}$ admits an open neighborhood $U$ in $\mathcal{H}$ and a $C^{k}$ smooth function $\rho: U \rightarrow \mathbb{R}$ satisfying the following conditions:

(i) $\Omega \cap U=\{\mathbf{z} \in U \mid \rho(\mathbf{z})<0\}$,

(ii) $\partial \Omega \cap U=\{\mathbf{z} \in U \mid \rho(\mathbf{z})=0\}$,

(iii) $U \backslash \bar{\Omega}=\{\mathbf{z} \in U \mid \rho(\mathbf{z})>0\}$ and

(iv) the differential $d \rho_{\mathbf{q}}$ is a non-zero functional for every $\mathbf{q} \in \partial \Omega \cap U$. 
The function $\rho$ is called a local defining function for $\Omega$ at $p$. The local defining function is not unique in general, but it is in effect unique since two given defining functions will differ only multiplicatively by a positive, smooth function.

Now we would like to define strong pseudoconvexity in the sense of Levi:

Definition 2.1. Consider the case in which the boundary point $\mathbf{p} \in \partial \Omega$ under consideration admits a $C^{2}$ local defining function $\rho$ defined on an open neighborhood $U$ of $\mathbf{p}$. Then we call the Hermitian form

$$
\Lambda \rho(\mathbf{p} ; \mathbf{v}, \mathbf{w}) \equiv D \bar{D} \rho(\mathbf{p} ; \mathbf{v}, \mathbf{w})
$$

the Levi form of $\rho$ at $\mathbf{p}$. We say that a point $\mathbf{p} \in \partial \Omega$ is a strongly pseudoconvex boundary point if there exist a $C^{2}$ smooth local defining function $\rho$ for $\Omega$ in a neighborhood of $\mathbf{p}$ and a constant $C>0$ satisfying the condition

$$
\Lambda \rho(\mathbf{p} ; \mathbf{v}, \mathbf{v}) \geq C\|\mathbf{v}\|^{2}, \forall \mathbf{v} \in \mathcal{H} .
$$

Note that this definition of strong pseudoconvexity is equivalent to requiring that $\mathbf{p} \in \partial \Omega$ admit an open neighborhood inside of which $\partial \Omega$ can be biholomorphically transformed to a strongly convex set. We will specify and use the precise meaning of this remark in what follows.

\section{MAIN THEOREM}

We now state the principal result of the present paper.

Theorem 3.1. Let $\Omega$ be a bounded convex domain in a separable Hilbert space $\mathcal{H}$. Assume that $\Omega$ admits a boundary point $\mathbf{p} \in \partial \Omega$ at which

(1) $\partial \Omega$ is $C^{2}$ smooth and strongly pseudoconvex in a neighborhood of $\mathbf{p}$, and

(2) there exist $\mathbf{q} \in \Omega$ and $f_{j} \in$ Aut $(\Omega)(j=1,2, \ldots)$ such that $f_{j}(\mathbf{q})$ converges to $\mathbf{p}$ in norm as $j \rightarrow \infty$.

Then $\Omega$ is biholomorphic to the unit ball $\mathbb{B}=\{z \in \mathcal{H} \mid\|\mathbf{z}\|<1\}$.

Theorem 3.1 is strongly analogous to the classical result of Rosay [ROS]. Its proof, however, is by no means a direct transliteration of Rosay's proof. To point out critical differences, we would like to mention first that the issues of localization in infinite dimensions are quite delicate and require new arguments. In addition, the holomorphic invariants based upon the classical volume elements are no longer valid. The geometry of Hilbert space presents some unfamiliar subtleties, even in the application of normal families of holomorphic mappings and of scaling methods.

\section{LOCALizATION}

It is a classical result of Cartan (see [NAR and references therein) that if a bounded domain $\Omega \subseteq \mathbb{C}^{n}$ has non-compact automorphism group, then there are a point $\mathbf{q} \in \Omega$ and a sequence of automorphisms $f_{j} \in \operatorname{Aut}(\Omega)$ such that $f_{j}(\mathbf{q}) \rightarrow \mathbf{p}$ for some $\mathbf{p} \in \partial \Omega$. The converse is true as well. Thus in practice, we think of non-compactness of the automorphism group, and non-compactness of an orbit, as interchangeable.

However, in the infinite-dimensional case, these two notions are not equivalent. For instance, the group of rotations (i.e. Hilbert space isometries) acts on the unit ball $\mathbb{B}$ in Hilbert space; it is non-compact, although it does not generate an orbit accumulating at a boundary point of $\mathbb{B}$. Several considerations (in light of the function theory and the geometric theory of bounded domains) lead us to believe 
that the existence of an automorphism orbit accumulating at a boundary point is a more essential condition than mere non-compactness of the automorphism group.

Thus we pose the following condition (which clearly plays a pivotal role in our formulation of Theorem 3.1):

$(\dagger)$ Let $\Omega$ be a bounded domain in $\mathcal{H}$ with a boundary point $\mathbf{p}$ near which $\partial \Omega$ is $C^{2}$ smooth and strongly pseudoconvex. We assume that there is a point $\mathbf{q} \in \Omega$ and a sequence of holomorphic automorphisms $f_{j} \in \operatorname{Aut}(\Omega)$ such that $f_{j}(\mathbf{q}) \rightarrow \mathbf{p}$ as $j$ tends to infinity.

Crucial to the analysis that we have just outlined is, at least in the finitedimensional case of the Wong-Rosay theorem, that one be able to localize the calculation of a certain invariant near $\mathbf{p}$. This invariant, denoted in the literature by $\mathcal{M}$, is the ratio between the Carathéodory and Kobayashi volume elements. More precisely, one wants to know that if $U$ is a small neighborhood of $\mathbf{p}$ in $\mathbb{C}^{n}$, then the calculation of $\mathcal{M}\left(f_{j}(\mathbf{q})\right)$ relative to $\Omega \cap U$ is essentially the same as the calculation of $\mathcal{M}\left(f_{j}(\mathbf{q})\right)$ relative to $\Omega$. In the infinite-dimensional case, the $\mathcal{M}$ invariant has no suitable definition and hence is no longer relevant. However, the localization argument which relates arbitrarily large subdomains to a local neighborhood of the orbit accumulation point is still quite essential.

The localization in the classical setting of finite dimensions involves delicate normal families arguments (see [KRA1]). In infinite dimensions it is considerably more subtle. This difficulty is exacerbated in part by the non-existence of a compact exhaustion of the domain.

We next present an effective version of localization in the infinite-dimensional setting.

We first need a suitable version of Montel's theorem in infinite dimensions. We present one now.

Lemma 4.1. Let $U$ be an open set in $\mathcal{H}$. Let $\mathcal{F}=\left\{f_{\alpha}\right\}_{\alpha \in \mathcal{A}}$ be a family of holomorphic (scalar-valued) functions on $U$. Assume that there is a constant $M>0$ such that $\left|f_{\alpha}(z)\right| \leq M$ for all $\alpha \in \mathcal{A}$ and all $z \in U$. Then each subsequence $\left\{f_{\alpha_{j}}\right\}$ has itself a subsequence $\left\{f_{\alpha_{j_{k}}}\right\}$ that converges uniformly on compact subsets of $U$.

Proof. Fix a compact set $K \subset U$. There is a positive number $\delta_{0}$ such that $\|k-u\| \geq$ $2 \delta_{0}$ for all $k \in K$ and $u \notin U$. Now for $k \in K$ and any unit vector $\eta \in \mathcal{H}$ we consider the disc $\mathcal{D}=\left\{k+\zeta \eta: \zeta \in \mathbb{C},|\zeta|<\delta_{0}\right\}$. Applying the standard one-variable Cauchy estimates on $\mathcal{D}$, we find that the directional derivative of any $f_{\alpha}$ at $k$ in the direction $\eta$ has a uniform bound-depending on $\delta_{0}$ and $M$, but not on $\alpha$. Therefore the elements of $\mathcal{F}$ satisfy a uniform Lipschitz estimate

$$
\left\|f_{\alpha}(z)-f_{\alpha}(z+h)\right\| \leq K \cdot\|h\|,
$$

where $K$ a constant independent of $\alpha$. As a result, the family $\mathcal{F}$ is equicontinuous. Of course it is equibounded by hypothesis.

As a result of these considerations, the Ascoli-Arzela theorem (which is valid on a compact metric space) applies and the required conclusion follows.

Remark. The Montel theorem that we have just formulated and proved will certainly suffice for our purposes. But it is worth noting that the following (formally) more general statement is now easily derived:

Let $U$ be an open set in $\mathcal{H}$. Let $\mathcal{F}=\left\{f_{\alpha}\right\}_{\alpha \in \mathcal{A}}$ be a family of holomorphic (scalar-valued) functions on $U$. Assume that for each compact set $K \subset$ 
$U$ there is a constant $M_{K}>0$ such that $\left|f_{\alpha}(z)\right| \leq M_{K}$ for all $\alpha \in \mathcal{A}$ and all $z \in M_{K}$. Then each subsequence $\left\{f_{\alpha_{j}}\right\}$ has itself a subsequence $\left\{f_{\alpha_{j_{k}}}\right\}$ that converges uniformly on compact subsets of $U$.

For a proof, we argue as follows (we are indebted to Laszlo Lempert for this elegant idea). We claim that each $P \in U$ has a neighborhood

$$
\mathcal{N}(P ; r) \equiv\{z \in \mathcal{H}:\|z-P\|<r\}
$$

on which the family $\mathcal{F}$ is uniformly bounded. If not, then inside each $\mathcal{N}(P ; 1 / n)$ there is a point $x_{n}$ and a function $f_{\alpha_{n}} \in \mathcal{F}$ such that $\left|f_{\alpha_{n}}\left(x_{n}\right)\right|>n$. Then

$$
L=\left\{x_{1}, x_{2}, \ldots\right\} \cup\{P\}
$$

is a compact set and $\mathcal{F}$ is unbounded on $K$. That is a contradiction. As a result, if $K$ is any compact set in $U$, then we can find a full neighborhood $W$ of $K$ on which $\mathcal{F}$ is uniformly bounded. Then Lemma 4.1, as stated, applies on $W$.

Now we have:

Lemma 4.2. Let $U$ be an open neighborhood of $\mathbf{p} \in \partial \Omega$ and assume that $\mathbf{x} \in \Omega$ satisfies $\lim _{j \rightarrow \infty} f_{j}(\mathbf{x})=\mathbf{p}$. Assume that $\Omega$ is strongly pseudoconvex in a neighborhood of $\mathbf{p}$. Then there exist a real number $r_{\mathbf{x}}>0$ and an integer $\nu_{\mathbf{x}}>0$ such that $f_{\nu}\left(\mathcal{N}\left(\mathbf{x} ; r_{\mathbf{x}}\right)\right) \subset U \cap \Omega$ for every $\nu>\nu_{\mathbf{x}}$.

Proof. Seeking a contradiction, we assume the contrary. This hypothesis in particular implies:

( $\ddagger$ There exists a subsequence $f_{j_{\nu}}$ of the sequence $f_{j}$ together with a point sequence $\mathbf{x}_{\nu}$ that converges to $\mathbf{x}$ in norm as $\nu \rightarrow \infty$ such that $f_{j_{\nu}}\left(\mathbf{x}_{\nu}\right) \notin U \cap \Omega$.

We shall denote the subsequence by $f_{\nu}$ for convenience.

Let $\left\{\mathbf{e}_{1}, \mathbf{e}_{2}, \ldots\right\}$ be an orthonormal basis for $\mathcal{H}$. In what follows, we think of an element $\mathbf{h} \in \mathcal{H}$ as having the form $\mathbf{h}=\sum_{j} z_{j} \mathbf{e}_{j}$, and we identify $\mathbf{h}$ with its coordinates $\left(z_{1}, z_{2}, \ldots\right)$. Now an analog of the finite-dimensional case allows us to consider a quadratic holomorphic polynomial mapping $G: \mathcal{H} \rightarrow \mathcal{H}$ which maps $U$ (an open neighborhood of $\mathbf{p}$ in $\mathcal{H}$ ) biholomorphically onto the open neighborhood $G(U)$ of $G(\mathbf{p})$ so that the following properties hold:

(1) $\Omega_{U} \equiv G(U \cap \Omega)$ is defined by the inequality

$$
\operatorname{Re} z_{1}>\psi\left(\left(\operatorname{Im} z_{1}\right) \mathbf{e}_{1}+\sum_{j=2}^{\infty} z_{j} \mathbf{e}_{j}\right) ;
$$

(2) $G(\mathbf{p})=\mathbf{0}$;

(3) $\Omega_{U}$ is supported by the closed subspace (a hyperplane) of codimension one defined by the equation $\operatorname{Re} z_{1}=0$;

(4) the function $\psi: G(U) \rightarrow \mathbb{R}$ is non-negative and strongly convex in the sense that it is $C^{2}$ smooth and there exists a constant $C>0$ satisfying

$$
d^{2} \psi(\mathbf{y} ; \mathbf{v}, \mathbf{v}) \geq C\|\mathbf{v}\|^{2} \text { for every } \mathbf{y} \in G(U) \text { and } \mathbf{v} \in \mathcal{H} .
$$

Notice that we may choose the neighborhood $U$ and the positive number $\epsilon$ so that the closure of the set

$$
V=\left\{\mathbf{z}=\sum_{j=1}^{\infty} z_{j} \mathbf{e}_{j} \in \Omega_{U} \mid \operatorname{Re} z_{1}<\epsilon\right\}
$$


is contained in $G(U)$. Now let

$$
\Sigma=\left\{\mathbf{z}=\sum_{j=1}^{\infty} z_{j} \mathbf{e}_{j} \in \bar{\Omega}_{U} \mid \operatorname{Re} z_{1}=\epsilon\right\} .
$$

Note that $\Sigma$ separates $\Omega_{U}$ into two disjoint subsets. Therefore $G^{-1}(\Sigma)$ also separates $U \cap \Omega$ into two disjoint subsets.

Now consider the straight line segment $\gamma_{\nu}$ joining $\mathbf{x}$ and $\mathbf{x}_{\nu}$. The segment $\gamma_{\nu}$ is contained in $\Omega$ for sufficiently large values of $\nu$, since $\mathbf{x}_{\nu}$ converges to $\mathbf{x}$ in norm as $\nu \rightarrow \infty$. Then the curve $f_{\nu} \circ \gamma_{\nu}$ connects $f_{\nu}(\mathbf{x})$ and $f_{\nu}\left(\mathbf{x}_{\nu}\right)$. For sufficiently large values of $\nu$, we have that $f_{\nu}(\mathbf{x}) \in\left(\left.G\right|_{U}\right)^{-1}(V)$ by hypothesis, and that $f_{\nu}\left(\mathbf{x}_{\nu}\right) \notin$ $\left(\left.G\right|_{U}\right)^{-1}(V)$ by $(\ddagger)$. In particular, the set

$$
\text { Image }\left(f_{\nu} \circ \gamma_{\nu}\right) \backslash\left(\left.G\right|_{U}\right)^{-1}(\Sigma)
$$

is disconnected. Therefore we can easily conclude by connectivity that there exists a point $\tilde{\mathbf{x}}_{\nu} \in \gamma_{\nu}$ such that $f_{\nu}\left(\tilde{\mathbf{x}}_{\nu}\right) \in\left(\left.G\right|_{U}\right)^{-1}(\Sigma)$. Notice that $\tilde{\mathbf{x}}_{\nu}$ also converges to $\mathbf{x}$ in norm.

Consider the sequence $G\left(f_{\nu}\left(\tilde{\mathbf{x}}_{\nu}\right)\right)$. This sequence consists of points in $\Sigma$. By the Banach-Alaoglu theorem, it has a subsequence that converges weakly. By an abuse of notation let us say that the sequence $G\left(f_{\nu}\left(\tilde{\mathbf{x}}_{\nu}\right)\right)$ converges to the point $\mathbf{y}^{*} \in \mathcal{H}$ weakly. Since $\Sigma$ is closed, bounded, and convex, we have $\mathbf{y}^{*} \in \Sigma$.

Now observe that, for an arbitrary $\mathbf{v} \in \mathcal{H}$, we have

$$
\begin{aligned}
-\left\langle\mathbf{y}^{*}, \mathbf{v}\right\rangle & =\left\langle 0-\mathbf{y}^{*}, \mathbf{v}\right\rangle \\
& =\lim _{\nu \rightarrow \infty}\left\langle G \circ f_{\nu}(\mathbf{x})-G \circ f_{\nu}\left(\tilde{\mathbf{x}}_{\nu}\right), \mathbf{v}\right\rangle .
\end{aligned}
$$

On the other hand, notice that the set $Y=\{\mathbf{x}\} \cup\left\{\mathbf{x}_{\nu} \mid \nu=1,2, \ldots\right\}$ is compact in the strong topology, since $\mathbf{x}_{\nu} \rightarrow \mathbf{x}$ in norm. Let $\ell_{\mathbf{v}}(\mathbf{z}) \equiv\langle\mathbf{z}, \mathbf{v}\rangle$. Then Lemma 4.1 implies that every subsequence of $\ell_{\mathbf{v}} \circ G \circ f_{\nu}$ has itself a subsequence that converges uniformly on the compact set $Y$. Consequently we have

$$
\left\langle\mathbf{y}^{*}, \mathbf{v}\right\rangle=0, \forall \mathbf{v} \in \mathcal{H} \text {. }
$$

However this is impossible, since $\mathbf{y}^{*} \in \Sigma$ and since $\Sigma$ does not contain the zero vector.

Next, for the strongly pseudoconvex boundary point $\mathbf{p}$ and the automorphisms $f_{j}$ of $\Omega$ as in $(\dagger)$, we show:

Lemma 4.3. Let $\mathbf{x}_{1}, \mathbf{x}_{2} \in \Omega$ be chosen such that there exists $r>0$ satisfying

$$
t \mathbf{x}_{1}+(1-t) \mathbf{x}_{2} \in \Omega, \quad \forall t \text { with }-r<t<1+r .
$$

If $\lim _{j \rightarrow \infty} f_{j}\left(\mathbf{x}_{1}\right)=\mathbf{p}$, then it follows that $\lim _{j \rightarrow \infty} f_{j}\left(\mathbf{x}_{2}\right)=\mathbf{p}$.

Proof. First of all, because of the strong pseudoconvexity, we may choose an open neighborhood $V$ with $\mathbf{p} \in V \subset U$ on which there exists a continuous local peak function $g: V \cap \bar{\Omega} \rightarrow \mathbb{C}$ satisfying

(A) $g$ is continuous on $V \cap \bar{\Omega}$,

(B) $g$ is holomorphic on $V \cap \Omega$,

(C) $g(\mathbf{p})=1$, and

(D) $|g(\mathbf{w})|<1$ for every $\mathbf{w} \in V \cap \bar{\Omega} \backslash\{\mathbf{p}\}$. 
Indeed, one directly imitates the construction and properties of the Levi polynomial $L_{P}(z)$ (see KRA1 p. 212]); then $\exp \left(L_{P}(z)\right)$ does the job.

We will shrink $U$ so that $U=V$ from here on. In particular, $g$ is now defined on $U$.

Let us write $D_{s}=\{z \in \mathbb{C}|| z \mid<s\}$ for each positive real number $s$, and let $D=D_{1}$ denote the open unit disc. Complexifying the straight line segment through $\mathbf{x}_{1}$ and $\mathbf{x}_{2}$, and then applying the Riemann mapping theorem if necessary, we have an injective holomorphic map $h: D_{(1+\delta)} \rightarrow \Omega$ such that $h(0)=\mathbf{x}_{1}$ and $h(\lambda)=\mathbf{x}_{2}$, for some $\lambda \in D$ and $\delta>0$.

The preceding lemma gives us an open neighborhood $W_{\mathbf{x}_{1}}$ of $\mathbf{x}_{1}$ in $\Omega$ such that there exists $N_{1}>0$ satisfying the condition $f_{j}\left(W_{\mathbf{x}_{1}}\right) \subset U \cap \Omega$ for all $j>N_{1}$. Note that there exists $\epsilon>0$ such that

$$
h\left(D_{\epsilon}\right) \subset W_{\mathbf{x}_{1}} .
$$

Now consider the sequence of mappings

$$
\left.g \circ f_{j} \circ h\right|_{D_{\epsilon}}: D_{\epsilon} \rightarrow D
$$

for $j=1,2, \ldots$ Notice that every subsequence has itself a subsequence that converges uniformly on compact subsets to a holomorphic mapping that has value 1 at 0 . Thus (by the maximum principle) the limit mapping is the constant mapping 1 , and hence the above sequence itself converges to the constant mapping 1, uniformly on compact subsets. In particular, we obtain that

$$
\lim _{j \rightarrow \infty} \sup _{z \in D_{r}}\left\|f_{j} \circ h(z)-\mathbf{p}\right\|=0
$$

for every $0<r<\epsilon$.

At this point, we will use the quadratic polynomial mapping $G: \mathcal{H} \rightarrow \mathcal{H}$ and the hyperplane $\Sigma$ used in the proof of the preceding lemma. Recall that $G$ maps $U \cap \Omega$ to a strongly convex set and $\Sigma$ separates $G(U \cap \Omega)$ into two disjoint subsets.

If $f_{j}\left(\mathbf{x}_{2}\right)$ does not converge to $\mathbf{p}$ in norm (as $j \rightarrow \infty$ ), then we may move $\Sigma$ closer to $\mathbf{p}$ if necessary and then choose a subsequence of $f_{j}\left(\mathbf{x}_{2}\right)$ (which, by an abuse of notation, we denote in the same way), so that $f_{j}\left(\mathbf{x}_{2}\right)$, for every $j$, belongs to the connected component of $G(U \cap \Omega) \backslash \Sigma$ which does not contain p. Therefore, considering the image of the straight line segment joining 0 and $\lambda$ in $D$, one obtains points $\xi_{j} \in D$ such that $G \circ f_{j} \circ h\left(\xi_{j}\right) \in \Sigma \cap \Omega$ for every $j$. Applying the BanachAlaoglu theorem again, we may assume (choosing a subsequence if necessary) that $G \circ f_{j} \circ h\left(\xi_{j}\right)$ converges weakly to $\mathbf{y}^{*}$. Again, the convexity implies that $\mathbf{y}^{*} \in \overline{\Sigma \cap \Omega}$.

Let $\mathbf{v} \in \mathcal{H}$, and write $\ell_{\mathbf{v}}(\mathbf{z})=\langle\mathbf{z}, \mathbf{v}\rangle$ for every $\mathbf{z} \in \mathcal{H}$. Consider the mappings

$$
\ell_{v} \circ G \circ f_{j} \circ h: D_{(1+\delta)} \rightarrow \mathbb{C}
$$

for $j=1,2, \ldots$ Notice that all their images are contained in the bounded domain $\ell_{v} \circ G(\Omega)$. Therefore Montel's theorem implies that a subsequence can be chosen (for which we use the same notation again) which converges uniformly on the closed disc $\bar{D}$. But we already know that $G \circ f_{j} \circ h$ converges to zero uniformly on $D_{\epsilon / 2}$. Therefore we see that $\ell_{v} \circ G \circ f_{j} \circ h$ converges uniformly on $\bar{D}$ to the zero function. In particular,

$$
\left\langle\mathbf{y}^{*}, \mathbf{v}\right\rangle=\lim _{j \rightarrow \infty}\left\langle G \circ f_{j} \circ h\left(\xi_{j}\right), \mathbf{v}\right\rangle=0 .
$$

Since $\mathbf{v} \in \mathcal{H}$ is arbitrary, we now have that $\mathbf{y}^{*}=\mathbf{0}$. However, this is absurd because $\Sigma$ does not contain the origin of $\mathcal{H}$. 
This contradiction yields the assertion.

Since $\Omega$ is a connected open subset of $\mathcal{H}$, it follows that every point of $\Omega$ can be joined to $\mathbf{q}$ in $(\dagger)$ by a polygonal line in $\Omega$ with finitely many break points. Therefore we immediately obtain the following

Corollary 4.4. Assuming $(\dagger)$ for $\Omega$, it follows that

$$
\lim _{j \rightarrow \infty} f_{j}(\mathbf{z})=\mathbf{p}
$$

for every $\mathbf{z} \in \Omega$.

Now we present the following strengthened form of the localization lemma.

Lemma 4.5. Let $\Omega$ be a bounded domain in $\mathcal{H}$ with a boundary point $\mathbf{p} \in \partial \Omega$ such that:

(1) $\partial \Omega$ is $C^{2}$ smooth and strongly pseudoconvex near $\mathbf{p}$, and

(2) there exist $\mathbf{q} \in \Omega$ and $f_{\ell} \in$ Aut $(\Omega)$ such that $f_{\ell}(\mathbf{q}) \rightarrow \mathbf{p}$ as $\ell \rightarrow \infty$ in norm.

Then there exists an increasing sequence of open subsets $\Omega_{1} \subset \Omega_{2} \subset \cdots$ of $\Omega$ satisfying:

(i) $\bigcup_{j=1}^{\infty} \Omega_{j}=\Omega$

(ii) for every open neighborhood $U$ of $\mathbf{p}$ in $\mathcal{H}$, there exists a subsequence $f_{\ell_{j}}$ of $f_{\ell}$ above such that $f_{\ell_{j}}\left(\Omega_{k}\right) \subset \Omega \cap U$ for every $j \geq k$.

Proof. We consider a countable dense subset (in the norm topology)

$$
Z=\left\{\mathbf{x}_{j} \in \Omega \mid j=1,2, \ldots\right\}
$$

of $\Omega$. For each $j$, the preceding lemmata allow us to choose, using the usual diagonalization process of selecting subsequences, a sequence of real numbers $r_{j} \equiv r_{x_{j}}>0$ and positive integers $\nu_{j}$ such that

$$
f_{\nu}\left(\mathcal{N}\left(\mathbf{x}_{j} ; r_{j}\right)\right) \subset U \cap \Omega, \forall \nu>\nu_{j} .
$$

Thus we set

$$
\ell_{j} \equiv \max \left\{\nu_{1}, \ldots, \nu_{j}\right\}+j \text { for each } j=1,2, \ldots
$$

so that

$$
\ell_{1}<\ell_{2}<\cdots
$$

and

$$
f_{\ell_{j}}\left(\mathcal{N}\left(\mathbf{x}_{k} ; r_{k}\right)\right) \subset U \quad \forall j \geq k .
$$

Now it suffices to let

$$
\Omega_{k} \equiv \bigcup_{\ell=1}^{k} \mathcal{N}\left(\mathbf{x}_{\ell} ; r_{\ell}\right)
$$

for each $k=1,2, \ldots$ This concludes the proof of the lemma as well as the construction of the localization process. 


\section{The Scaling Process}

We now discuss the scaling method (originated by S. Pinchuk in $\mathbb{C}^{n}$; see IK1] for the history) and how it should be adjusted so that it is a useful technique in the study of automorphisms of bounded domains in infinitely many dimensions.

For this purpose, we continue to work with an orthonormal basis $\mathbf{e}_{1}, \mathbf{e}_{2}, \ldots$ for the separable Hilbert space $\mathcal{H}$ under consideration.

5.1. Normalization at the strongly pseudoconvex point. We continue our exposition with the notation above; let $\Omega$ be a bounded domain in $\mathcal{H}$ with a $C^{2}$ strongly pseudoconvex boundary point $\mathbf{p}$ at which the automorphism orbit $f_{j}(\mathbf{q})$ accumulates.

Furthermore, throughout the rest of the paper we utilize the following notation: for each $\mathbf{z}=\sum_{j=1}^{\infty} z_{k} \mathbf{e}_{k} \in \mathcal{H}$, we set

$$
\mathbf{z}^{\prime}=\sum_{j=2}^{\infty} z_{k} \mathbf{e}_{k} .
$$

As before, let $U$ be an open neighborhood of $\mathbf{p}$ and let $G: U \rightarrow \mathcal{H}$ be the quadratic change of coordinates which maps $U$ biholomorphically onto $G(U)$ in such a way that

- $G(\mathbf{p})=\mathbf{0}$, where $\mathbf{0}$ denotes the origin of $\mathcal{H}$, and

- $\Omega_{U} \equiv G(\Omega \cap U)$ is represented by

$$
\Omega_{U}=\left\{\mathbf{z}=\sum_{j=1}^{\infty} z_{k} \mathbf{e}_{k} \in G(U) \mid \operatorname{Re} z_{1}>\psi\left(\left(\operatorname{Im} z_{1}\right) \mathbf{e}_{1}+\mathbf{z}^{\prime}\right)\right\}
$$

where $\psi: \mathcal{H} \rightarrow \mathbb{R}$ is a $C^{2}$ function satisfying

$\circ \psi(\mathbf{0})=0$;

$\circ d \psi(\mathbf{0} ; \mathbf{v})=0$ for every $\mathbf{v} \in \mathcal{H}$;

$\circ$ there exists a constant $C_{0}>0$ such that

$$
d^{2} \psi(\mathbf{x} ; \mathbf{v}, \mathbf{v}) \geq C_{0}\|\mathbf{v}\|^{2}, \forall \mathbf{x} \in G(U), \forall \mathbf{v} \in \mathcal{H} .
$$

We write

$$
\rho_{U}(\mathbf{z})=-\operatorname{Re} z_{1}+\psi\left(\left(\operatorname{Im} z_{1}\right) \mathbf{e}_{1}+\mathbf{z}^{\prime}\right),
$$

which is the defining function of $\Omega_{U}$ in $G(U)$; we are going to use $\rho_{U}$ repeatedly in the arguments below.

5.2. Centering of the automorphism orbit. Let us fix the notation for the automorphism orbit: put

$$
\mathbf{q}_{\nu} \equiv G \circ f_{\nu}(\mathbf{q}) \text { for } \nu=1,2, \ldots .
$$

(We may need to pass to a subsequence of $f_{\nu}$ when appropriate.)

Consider the sequence of points $\mathbf{q}_{\nu} \in \Omega_{U}$ approaching the origin $\mathbf{0}$ as $\nu \rightarrow \infty$. First of all, we write

$$
\mathbf{q}_{\nu}=\sum_{j=1}^{\infty} q_{\nu j} \mathbf{e}_{j}, q_{\nu j} \in \mathbb{C}
$$


Then, for each $\nu=1,2, \ldots$, we consider the boundary point

$$
\mathbf{p}_{\nu} \equiv q_{\nu 1}^{*} \mathbf{e}_{1}+\mathbf{q}_{\nu}^{\prime} .
$$

Here $q_{\nu 1}^{*} \in \mathbb{C}$ is chosen to satisfy the relations

$$
\mathbf{p}_{\nu} \in \partial \Omega_{U} \text { and } q_{\nu 1}-q_{\nu 1}^{*}>0
$$

for each $\nu=1,2, \ldots$

Then we apply the complex affine linear isomorphism $\Psi_{\nu}: \mathbf{z} \mapsto \mathbf{w}: \mathcal{H} \rightarrow \mathcal{H}$ defined by

$$
\begin{aligned}
& w_{1}=e^{i \theta_{\nu}}\left(z_{1}-q_{\nu 1}^{*}\right)+T_{\nu}\left(\mathbf{z}^{\prime}-\mathbf{q}_{\nu}^{\prime}\right), \\
& \mathbf{w}^{\prime}=\mathbf{z}^{\prime}-\mathbf{q}_{\nu}^{\prime},
\end{aligned}
$$

where the number $\theta_{\nu} \in \mathbb{R}$ and the linear functional $T_{\nu}: \mathcal{H} \rightarrow \mathbb{C}$ are to be chosen to satisfy the following conditions:

- The transformed domain $\Psi_{\nu}\left(\Omega_{U}\right)$ is supported by the hyperplane defined by $\operatorname{Re} w_{1}=0$, and

- $\Psi_{\nu}\left(\Omega_{U}\right) \subset\left\{\mathbf{w}=\sum_{j} w_{j} \mathbf{e}_{j} \mid \operatorname{Re} w_{1}>0\right\}$.

These conditions are easily satisfied if we choose $\theta_{\nu}$ and $T_{\nu}$ as specified below. Recall that $\rho_{U}=-\operatorname{Re} w_{1}+\psi\left(\operatorname{Im} w_{1}, \mathbf{w}^{\prime}\right)$ is the defining function for $\Omega_{U}$. Our choices for $\theta_{\nu}$ and $T_{\nu}$ are determined by the following equations:

$$
\begin{aligned}
\theta_{\nu} & =\tan ^{-1}\left(\frac{\partial \psi}{\partial\left(\operatorname{Re} w_{1}\right)}\right) \\
T_{\nu}\left(\sum_{k=2}^{\infty} v_{k} \mathbf{e}_{k}\right) & =2 e^{i \theta_{\nu}} \sum_{k=2}^{\infty} \frac{\partial \psi}{\partial w_{k}}\left(\operatorname{Im} q_{\nu 1}^{*}, \mathbf{q}_{\nu}^{\prime}\right) v_{k}
\end{aligned}
$$

and

$$
T_{\nu}\left(\mathbf{e}_{1}\right)=0 .
$$

Notice that $T_{\nu}$ is a bounded linear functional on $\mathcal{H}$.

In conclusion, we have achieved that $T_{\nu}\left(\Omega_{U}\right)$ satisfies the following properties (for every $\nu=1,2, \ldots$ ):

(C1) $\Psi_{\nu}\left(\Omega_{U}\right)$ is supported by the real hyperplane defined by $\operatorname{Re} w_{1}=0$ at the origin $\mathbf{0}$ in $\mathcal{H}$;

(C2) $\mathbf{0} \in \partial\left(\Psi_{\nu}\left(\Omega_{U}\right)\right)$;

(C3) $\Psi_{\nu}\left(\mathbf{q}_{\nu}\right)=e^{i \theta_{\nu}}\left(q_{\nu 1}-q_{\nu_{1}}^{*}\right) \mathbf{e}_{1}$.

Also, notice that $e^{i \theta_{\nu}}$ converges to 1 as $\nu$ tends to $\infty$.

5.3. The scaling map for $\Omega$ along $f_{\nu}$. Now we consider the linear isomorphism $L_{\nu}: \mathcal{H} \rightarrow \mathcal{H}$ defined by

$$
L_{\nu}(\mathbf{w})=\frac{w_{1} \mathbf{e}_{1}}{\lambda_{\nu}}+\frac{\mathbf{w}^{\prime}}{\sqrt{\lambda_{\nu}}}
$$

for each $\nu$, where

$$
\lambda_{\nu}=q_{\nu 1}-q_{\nu 1}^{*} .
$$

The scaling process along $\mathbf{q}_{\nu}$ consists of the sequence of maps defined by

$$
\sigma_{\nu} \equiv L_{\nu} \circ \Psi_{\nu} \circ G \circ f_{\nu}: \Omega_{\nu} \rightarrow \mathcal{H}, \text { for } \nu=1,2, \ldots
$$


In what follows, we shall develop properties of the scaling process, and we also shall implement several refinements.

\section{Initial Scaling at a Strongly Pseudoconvex Point}

The goal of this section is to show that the scaling sequence $\left\{\sigma_{\nu} \mid \nu=1,2, \ldots\right\}$ admits a subsequence that converges to a mapping that maps $\Omega$ biholomorphically onto a domain of $\mathcal{H}$ which is in turn biholomorphic to the unit ball in $\mathcal{H}$.

6.1. Ellipsoidal envelopes. Let us return to $\Omega_{U}$ with the defining function $\rho_{U}(\mathbf{z})$ $=-\operatorname{Re} z_{1}+\psi\left(\left(\operatorname{Im} z_{1}\right) \mathbf{e}_{1}+\mathbf{z}^{\prime}\right)$ which is $C^{2}$ smooth at every point of $G(U)$; the defining function admits a constant $C_{0}>0$ satisfying the condition

$$
d^{2} \rho_{U}(\mathbf{0} ; \mathbf{v}, \mathbf{v}) \geq C_{0}\|\mathbf{v}\|^{2} \quad \forall \mathbf{v} \in \mathcal{H} .
$$

Recall the centering map $\Psi_{\nu}$ from Section 5.2 associated with the point sequence $\mathbf{q}_{\nu}=G \circ f_{\nu}(\mathbf{q})$. Let us put

$$
\begin{aligned}
\rho_{\nu}(\mathbf{z}) & \equiv \rho \circ \Psi_{\nu}^{-1}(\mathbf{z}), \\
\Phi_{\nu}^{-1}(\mathbf{z}) & \equiv \Psi_{\nu}^{-1}(\mathbf{z})-\mathbf{p}_{\nu} .
\end{aligned}
$$

Notice that $d \Psi_{\nu}^{-1}(\mathbf{x} ; \mathbf{z})=\Phi_{\nu}^{-1}(\mathbf{z})$ for all $\mathbf{z} \in \mathcal{H}$, regardless of the location of $\mathbf{x}$ in $\mathcal{H}$. See the previously defined notation (Subsection 5.2) for $\mathbf{p}_{\nu}$ as well as the definition of the centering map $\Psi_{\nu}$. Now a direct computation yields

$$
\begin{aligned}
d \rho_{\nu}(\mathbf{q} ; \mathbf{v}) & =d \rho\left(\Psi_{\nu}^{-1}(\mathbf{q}) ; \Phi_{\nu}^{-1}(\mathbf{v})\right) \\
d^{2} \rho_{\nu}(\mathbf{0} ; \mathbf{v}, \mathbf{v}), & =d^{2} \rho\left(\mathbf{p}_{\nu} ; \Phi_{\nu}^{-1}(\mathbf{v}), \Phi_{\nu}^{-1}(\mathbf{v})\right) .
\end{aligned}
$$

Then we see that

$$
\begin{aligned}
& \frac{1}{\|\mathbf{z}\|^{2}}\left[\rho_{\nu}(\mathbf{z})-\rho_{\nu}(\mathbf{0})-d \rho_{\nu}(\mathbf{0} ; \mathbf{z})\right] \\
= & \frac{\rho_{U}\left(\Psi_{\nu}^{-1}(\mathbf{z})\right)-\rho_{U}\left(\Psi_{\nu}^{-1}(\mathbf{0})\right)-d \rho_{U}\left(\Psi_{\nu}^{-1}(\mathbf{0}) ; \Phi_{\nu}^{-1}(\mathbf{z})\right)}{\left\|\Phi_{\nu}^{-1}(\mathbf{z})\right\|^{2}} \cdot \frac{\left\|\Phi_{\nu}^{-1}(\mathbf{z})\right\|^{2}}{\|\mathbf{z}\|^{2}} .
\end{aligned}
$$

Recall that the sequence $\Psi_{\nu}^{-1}$ converges to the identity map of $\mathcal{H}$ uniformly on every bounded subset of $\mathcal{H}$ and that $\rho_{U}$ is $C^{2}$ smooth. Notice that we may choose $N>0$ and $r>0$ such that

$$
\left\|\Phi_{\nu}^{-1}(\mathbf{z})\right\| \geq \frac{1}{2}\|\mathbf{z}\|, \quad \forall \mathbf{z} \in \mathcal{H} \text { with }\|\mathbf{z}\| \leq r \text { and } \forall \nu>N .
$$

Then, shrinking $r>0$ if necessary, we see that the $C^{2}$ smoothness of $\rho_{U}$ implies that

$$
\frac{\rho_{U}\left(\Psi_{\nu}^{-1}(\mathbf{z})\right)-\rho_{U}\left(\Psi_{\nu}^{-1}(\mathbf{0})\right)-d \rho_{U}\left(\Psi_{\nu}^{-1}(\mathbf{0}) ; \Phi_{\nu}^{-1}(\mathbf{z})\right)}{\left\|\Phi_{\nu}^{-1}(\mathbf{z})\right\|^{2}} \cdot \frac{\left\|\Phi_{\nu}^{-1}(\mathbf{z})\right\|^{2}}{\|\mathbf{z}\|^{2}}
$$

converges to

$$
d^{2} \rho_{U}\left(\mathbf{0} ; \frac{\mathbf{z}}{\|\mathbf{z}\|}, \frac{\mathbf{z}}{\|\mathbf{z}\|}\right)
$$

uniformly on $\mathcal{N}(\mathbf{0} ; r)$ in $\mathcal{H}$ as $\nu$ tends to infinity. Therefore we are able to deduce the following: 
Lemma 6.1. There exist positive constants $r, N$, and $c$ satisfying the inequality

$$
\rho_{\nu}(\mathbf{z})-\rho_{\nu}(\mathbf{0})-d \rho_{\nu}(\mathbf{0} ; \mathbf{z})>c\|\mathbf{z}\|^{2}
$$

for every $\mathbf{z} \in \mathcal{H}$ with $\|\mathbf{z}\|<r$ and for every $\nu>N$.

6.2. First adjustment of the scaling sequence. Recall that the domain $L_{\nu} \circ$ $\Psi_{\nu}\left(\Omega_{U}\right)$ is defined by the inequality

$$
c_{\nu} \operatorname{Re} z_{1}>d^{2} \rho_{\nu}\left(\mathbf{0} ; \mathbf{z}^{\prime}, \mathbf{z}^{\prime}\right)+R_{\nu}(\mathbf{z}),
$$

where:

- $c_{\nu}$ converges to 1 ;

- $R_{\nu}(\mathbf{z})$ tends to zero uniformly on each bounded set

as $\nu$ tends to $\infty$.

For each sufficiently large $\nu$, we consider the linear transformation $S_{\nu}: \mathcal{H} \rightarrow \mathcal{H}$ satisfying

- $S_{\nu}\left(\mathbf{e}_{1}\right)=\mathbf{e}_{1} / c_{\nu}$

- $S_{\nu}\left(\left(\mathbf{e}_{1}\right)^{\perp}\right) \subseteq\left(\mathbf{e}_{1}\right)^{\perp}$, and

- $d^{2} \rho_{\nu}\left(\mathbf{0} ; S_{\nu}^{-1}\left(\mathbf{z}^{\prime}\right), S_{\nu}^{-1}\left(\mathbf{z}^{\prime}\right)\right)=\left\langle\mathbf{z}^{\prime}, \mathbf{z}^{\prime}\right\rangle_{\mathcal{H}}$.

Notice that we can choose $S_{\nu}$ so that both $S_{\nu}$ and $S_{\nu}^{-1}$ are uniformly bounded linear operators on $\mathcal{H}$.

Notice that there exists $r>0$ such that

$$
\left\langle S_{\nu}^{-1}\left(\mathbf{z}^{\prime}\right), S_{\nu}^{-1}\left(\mathbf{z}^{\prime}\right)\right\rangle \geq r\left\langle\mathbf{z}^{\prime}, \mathbf{z}^{\prime}\right\rangle, \quad \forall \mathbf{z}^{\prime} \in\left(\mathbf{e}_{1}\right)^{\perp},
$$

for every $\nu=1,2, \ldots$.

Therefore we have the following:

(i) $S_{\nu} \circ L_{\nu} \circ \Psi_{\nu}\left(\Omega_{\nu}\right)$ is defined by

$$
\left.\operatorname{Re} z_{1}\right\rangle\left\langle\mathbf{z}^{\prime}, \mathbf{z}^{\prime}\right\rangle+R_{\nu}(\mathbf{z}),
$$

where $R_{\nu}$ converges to zero uniformly on every bounded subset of $\mathcal{H}$, as $\nu \rightarrow \infty$.

(ii) $S_{\nu} \circ L_{\nu} \circ \Psi_{\nu}\left(\Omega_{U}\right) \subset \mathcal{E}(1, r)$ for some fixed $r>0$ independent of $\nu$, where

$$
\mathcal{E}(1, r)=\left\{\mathbf{z} \in \mathcal{H} \mid \operatorname{Re} z_{1}>r\left\langle\mathbf{z}^{\prime}, \mathbf{z}^{\prime}\right\rangle\right\} .
$$

Then we consider the linear fractional transformation $F: \mathcal{H} \rightarrow \mathcal{H}$ defined by

$$
F(\mathbf{z})=\left(\frac{z_{1}-1}{z_{1}+1}\right) \mathbf{e}_{1}+\frac{2}{z_{1}+1} \sum_{k=2}^{\infty} z_{k} \mathbf{e}_{k}
$$

This mapping transforms $\mathcal{E}(1, r)$ biholomorphically onto $\mathbb{B}_{r} \equiv\left\{\left.\mathbf{z} \in \mathcal{H}|| z_{1}\right|^{2}+\right.$ $\left.r\left\|\mathbf{z}^{\prime}\right\|^{2}<1\right\}$.

Finally, we write

$$
\begin{aligned}
\omega_{\nu} & \equiv F \circ S_{\nu} \circ \sigma_{\nu} \\
& =F \circ S_{\nu} \circ L_{\nu} \circ \Psi_{\nu} \circ G \circ f_{\nu}
\end{aligned}
$$

for each $\nu=1,2, \ldots$ The map $\omega_{\nu}$ sends $\Omega_{\nu}$ into $\mathbb{B}_{r}$ for every $r>1$. 
6.3. Convergence of the first adjustment. We first specify the domains $\Omega_{\nu}$ which exhaust $\Omega$.

Let us consider

$$
\Sigma_{n}=\mathbb{C e}_{1} \oplus \cdots \oplus \mathbb{C e}_{n}, \quad n=1,2, \ldots .
$$

Let $Z_{k}$ be a countable dense subset of $\Sigma_{k}$ for each $k=1,2, \ldots$, and let

$$
Z=\bigcup_{k=1}^{\infty} Z_{k} .
$$

Let us write $Z=\left\{\mathbf{x}_{1}, \mathbf{x}_{2}, \ldots\right\}$.

Then, as in the localization process introduced in Section 4 , we may choose the balls $\mathcal{N}\left(\mathbf{x}_{j} ; r_{j}\right)$ and define

$$
\Omega_{\ell}=\bigcup_{j=1}^{\ell} \mathcal{N}\left(\mathbf{x}_{j} ; r_{j}\right)
$$

for each $\ell=1,2, \ldots$.

Let $r>0$ be chosen arbitrarily. Then notice that the steps (i) and (ii) of Subsection 6.2 will be valid for $\mathcal{E}(1, r)$ if we choose $U$ sufficiently small. Therefore the localization lemma in Section 4 allows us to choose a subsequence so that

$$
\omega_{\nu}\left(\Omega_{\nu}\right) \subset \mathbb{B}_{r}
$$

for every $\nu=1,2, \ldots$

Now the Banach-Alaoglu theorem allows us to choose a subsequence $\omega_{\nu_{k}}$ of $\omega_{\nu}$ such that $\omega_{\nu_{k}}\left(\mathbf{x}_{1}\right)$ converges weakly to $\widehat{\omega}\left(\mathbf{x}_{1}\right)$ as $\nu \rightarrow \infty$. Then we can inductively choose a subsequence $\left\{\omega_{\nu_{k}}\right\}$ of $\omega_{\nu_{k-1}}$ so that $\omega_{\nu_{k}}\left(\mathbf{x}_{j}\right)$ converges weakly to $\widehat{\omega}\left(\mathbf{x}_{j}\right)$ as $\nu \rightarrow \infty$ for every $j=1,2, \ldots, k$.

Re-naming the sequence $\omega_{\nu}=\omega_{\nu_{\nu}}$, we see that

$$
\omega_{\nu}(\mathbf{x}) \rightarrow \widehat{\omega}(\mathbf{x}) \text { weakly, as } \nu \rightarrow \infty
$$

for every $\mathrm{x} \in Z$.

Let $\ell \in \mathcal{H}^{*}$. Consider the sequence

$$
\left.\ell \circ \omega_{\nu}\right|_{\Sigma_{n} \cap \Omega_{\nu}}: \Sigma_{n} \cap \Omega_{\nu} \rightarrow \mathbb{C} .
$$

Notice that this is a uniformly bounded sequence. By Montel's theorem, there exists a subsequence $\ell \circ \omega_{\nu_{k}}$ that converges to the holomorphic function, say $\phi$ : $\Sigma_{n} \cap \Omega \rightarrow \mathbb{C}$, uniformly on compact subsets of $\Sigma_{n} \cap \Omega$. Notice that for each $\mathbf{x} \in Z_{n}$ we have

$$
\begin{aligned}
& |\phi(\mathbf{x})-\ell \circ \widehat{\omega}(\mathbf{x})| \\
& \quad \leq \quad\left|\phi(\mathbf{x})-\ell \circ \omega_{\nu_{k}}(\mathbf{x})\right|+\left|\ell \circ \omega_{\nu_{k}}(\mathbf{x})-\ell \circ \widehat{\omega}(\mathbf{x})\right| \\
& \quad 0
\end{aligned}
$$

as $k \rightarrow \infty$. Therefore $\ell \circ \widehat{\omega}=\phi$ on $Z_{n}$. In particular, $\ell \circ \widehat{\omega}$ extends uniquely to a holomorphic function on $\Sigma_{n} \cap \Omega$, which we will denote with the same notation.

Now we prove

Lemma 6.2. The sequence $\omega_{\nu}(\mathbf{z})$ converges weakly to an element $\widehat{\omega}(\mathbf{z})$ for every $\mathbf{z} \in \Sigma_{n} \cap \Omega$. 
Proof. Recall that $\omega_{\nu}$ forms an equibounded family of holomorphic mappings. Therefore the Banach-Alaoglu theorem allows us to choose a subsequence $\omega_{\nu_{k}}$ such that

$$
\omega_{\nu_{k}}(\mathbf{z}) \rightarrow \alpha_{\mathbf{z}} \in \mathcal{H} \text { weakly }
$$

as $k \rightarrow \infty$.

Let $\ell \in \mathcal{H}^{*}$. Applying Montel's theorem to the restriction of these maps to $\Sigma_{n} \cap \Omega$, we may again choose a subsequence $\omega_{\nu_{j_{k}}}$ such that

$$
\ell \circ \omega_{\nu_{j_{k}}}(\mathbf{z}) \rightarrow \psi(\mathbf{z})
$$

uniformly on compact subsets of $\Sigma_{n} \cap \Omega$. Notice that we then obtain

$$
\psi(\mathbf{x})=\ell \circ \widehat{\omega}(\mathbf{x}) \text { for every } \mathbf{x} \in Z_{n}
$$

by the argument preceding the statement of this lemma. Therefore we can easily deduce that $\psi(\mathbf{z})$ is indeed uniquely determined to be $\ell \circ \widehat{\omega}(\mathbf{z})$, for every $\ell \in \mathcal{H}^{*}$. Consequently we have

$$
\alpha_{\mathbf{z}}=\sum_{k=1}^{\infty} \ell_{k} \circ \widehat{\omega}(\mathbf{z}) \mathbf{e}_{k}
$$

where $\ell_{k}(\mathbf{w})=\left\langle\mathbf{w}, \mathbf{e}_{k}\right\rangle$ for every $\mathbf{w} \in \mathcal{H}$.

Hence it suffices to let $\widehat{\omega}(\mathbf{z})=\alpha_{\mathbf{z}}$ for each $\mathbf{z} \in \Sigma_{n} \cap \Omega$.

Note that the lemma defines $\widehat{\omega}$ on $\Sigma_{n} \cap \Omega$. Since $\ell \circ \widehat{\omega}$ is a holomorphic function on $\Sigma_{n} \cap \Omega$ for every $\ell \in \mathcal{H}^{*}$, Hartogs's analyticity theorem implies:

Lemma 6.3. The restriction $\left.\widehat{\omega}\right|_{\Sigma_{n} \cap \Omega}: \Sigma_{n} \cap \Omega \rightarrow \mathcal{H}$ is a holomorphic mapping for every finite-dimensional slice $\Sigma_{n} \cap \Omega$ of $\Omega$.

Now we extend $\widehat{\omega}$ to a holomorphic mapping of $\Omega$ into $\mathcal{H}$. Let

$$
\mathbf{z}=\sum_{k=1}^{\infty} z_{k} \mathbf{e}_{k} \in \Omega
$$

We write

$$
\mathbf{z}_{n}=\sum_{k=1}^{n} z_{k} \mathbf{e}_{k}
$$

for every $n=1,2, \ldots$. Note that $\mathbf{z}_{n} \in \Sigma_{n} \cap \Omega$, and $\mathbf{z}_{n} \rightarrow \mathbf{z}$ in norm as $n \rightarrow \infty$.

Let $Q \equiv\{\mathbf{z}\} \cup\left\{\mathbf{z}_{n} \mid n=1,2, \ldots\right\}$. Let $\ell \in \mathcal{H}^{*}$ be arbitrarily given. Since $Q$ is compact, it follows that a subsequence $\omega_{\nu_{j}}$ of $\omega_{\nu}$ can be chosen so that $\ell \circ \omega_{\nu_{j}}$ converges to $\ell \circ \widehat{\omega}$, uniformly on $Q$. Therefore it follows immediately that the sequence $\ell \circ \widehat{\omega}\left(\mathbf{z}_{n}\right)$ is uniformly Cauchy. Thus it converges - since $\mathcal{H}$ is complete. Let us write

$$
\beta_{k} \equiv \lim _{n \rightarrow \infty}\left\langle\widehat{\omega}\left(\mathbf{z}_{n}\right), \mathbf{e}_{k}\right\rangle
$$

for each $k=1,2, \ldots$ Then we define

$$
\widehat{\omega}(\mathbf{z})=\sum_{k=1}^{\infty} \beta_{k} \mathbf{e}_{k} .
$$

This allows us to extend $\widehat{\omega}$ to all of $\Omega$.

Now the question we need to resolve is whether the mapping $\widehat{\omega}$ so defined is a holomorphic mapping of $\Omega$ into $\mathcal{H}$. To answer this question we have: 
Proposition 6.4. The mapping $\widehat{\omega}: \Omega \rightarrow \mathcal{H}$ is holomorphic, and $\widehat{\omega}(\Omega) \subset \mathbb{B}$.

Proof. Since

$$
\begin{aligned}
\left|\ell \circ \omega_{\nu}(\mathbf{z})-\ell \circ \widehat{\omega}(\mathbf{z})\right| \leq & \left|\ell \circ \omega_{\nu}(\mathbf{z})-\ell \circ \omega_{\nu}\left(\mathbf{z}_{n}\right)\right| \\
& +\left|\ell \circ \omega_{\nu}\left(\mathbf{z}_{n}\right)-\ell \circ \widehat{\omega}\left(\mathbf{z}_{n}\right)\right|+\left|\ell \circ \widehat{\omega}\left(\mathbf{z}_{n}\right)-\ell \circ \widehat{\omega}(\mathbf{z})\right|,
\end{aligned}
$$

we can deduce immediately that $\omega_{\nu}$ converges weakly to $\widehat{\omega}$ at every point of $\Omega$.

Now consider the $\mathcal{H}$-valued mapping

$$
g(\lambda) \equiv \widehat{\omega}(\mathbf{p}+\lambda \mathbf{v})
$$

of $\lambda \in \mathbb{C}$, where $\mathbf{p} \in \Omega$ and $\mathbf{v} \in \mathcal{H}$. The function $g$ is well-defined for all $\lambda \in \mathbb{C}$ with $|\lambda|<r$ for some sufficiently small $r>0$.

Let us consider the projections $\pi_{n}: \mathcal{H} \rightarrow \mathbb{C e}_{1} \oplus \ldots \oplus \mathbb{C e}_{n}$ for each $n=1,2, \ldots$ and examine the mapping

$$
g_{n}(\lambda) \equiv \widehat{\omega}\left(\pi_{n}(\mathbf{p})+\lambda \pi_{n}(\mathbf{v})\right),
$$

which is well-defined for all $\lambda \in \mathbb{C}$ with $|\lambda|<r$. (Shrink $r>0$ if necessary. But note that one may choose $r$ independent of $n$.)

Lemma 6.3 implies that $g_{n}$ is holomorphic for every $n$, and $\left\{g_{n}\right\}$ forms an equibounded family. Therefore, for each $\ell \in \mathcal{H}^{*}, \ell \circ g_{n}$ admits a subsequence, say $\ell \circ g_{n_{j}}$, that converges to a holomorphic function, say $h$, uniformly on compact subsets. On the other hand, the preceding arguments yield that $\ell \circ g_{n}(\lambda)=\ell \circ \widehat{\omega}\left(\pi_{n}(\mathbf{p}+\lambda \mathbf{v})\right)$ converges to $\ell \circ \widehat{\omega}(\mathbf{p}+\lambda \mathbf{v})$ pointwise, by construction. Therefore we see that

$$
\ell \circ \widehat{\omega}(\mathbf{p}+\lambda \mathbf{v})=h(\lambda)
$$

for every $\lambda \in \mathbb{C}$ with $|\lambda|<r$. Since $\ell \in \mathcal{H}^{*}$ is arbitrary, we obtain that $\widehat{\omega}(\mathbf{p}+\lambda \mathbf{v})$ is a holomorphic mapping of $\lambda$ for every fixed $\mathbf{p} \in \Omega$ and $\mathbf{v} \in \mathcal{H}$.

Notice that the weak limit of a sequence in $\mathcal{H}$ stays in the closed convex hull of the given sequence. Therefore we note that the set $\widehat{\omega}(\Omega)$ is bounded. Altogether, the mapping $\widehat{\omega}: \Omega \rightarrow \mathcal{H}$ is bounded, and holomorphic on every one-dimensional slice (and hence in every finite-dimensional slice by Hartogs's analyticity theorem). Consequently, we may conclude that $\widehat{\omega}: \Omega \rightarrow \mathcal{H}$ is holomorphic. (Notice that the Gateaux definition of holomorphicity formally requires $C^{1}$ smoothness, but the local boundedness and analyticity in every finite-dimensional slice give sufficient grounds for holomorphicity of the function. See for instance $\mathrm{Mu}$.)

Now we repeat that one may adjust the subsequence $\omega_{\nu}$ (choosing a subsequence if necessary) so that $\omega_{\nu}\left(\Omega_{\nu}\right) \subset\left(1+\frac{1}{\nu}\right) \mathbb{B}$. We have addressed this point in Section 6.3 above, in which the localization lemma was essential.

Using again the fact that the weak limits stay in the closed convex hull of the sequence in consideration, we obtain finally that

$$
\widehat{\omega}(\Omega) \subset \overline{\mathbb{B}} \text {. }
$$

Recall, by definition, that $\omega_{\nu}(\mathbf{q})=\mathbf{0}$ for every $\nu=1,2, \ldots$ Thus $\widehat{\omega}(\mathbf{q})=\mathbf{0}$. Then we may apply the Maximum Principle as in the finite-dimensional case to conclude immediately that $\widehat{\omega}(\Omega) \subset \mathbb{B}$. This completes the proof.

It is also true that the same principle as in the proof of Proposition 6.4 yields a holomorphic weak limit mapping of the inverse maps of the elements of the above scaling sequence. Just the same, it is not yet enough to conclude that the scaled limit mapping so constructed above is a biholomorphism. So we shall adjust the scaling process again in the next section. 


\section{Second Adjustment of Scaling}

We begin this section with some functional-analytic arguments.

7.1. Base-change with uniformly bounded operators. Consider a sequence $T_{\nu}: \mathcal{H} \rightarrow \mathcal{H}$ of invertible $\mathbb{C}$-linear operators admitting a constant $C>0$ such that

$$
\frac{1}{C}\|\mathbf{v}\| \leq\left\|T_{\nu}(\mathbf{v})\right\| \leq C\|\mathbf{v}\| \text { and } \frac{1}{C}\|\mathbf{v}\| \leq\left\|T_{\nu}^{-1}(\mathbf{v})\right\| \leq C\|\mathbf{v}\|
$$

for every $\mathbf{v} \in \mathcal{H}$ and $\nu=1,2, \ldots$ In general, we only have the subsequential weak convergence for a sequence of such operators. The momentary goal is to improve its convergence by composing with Hilbert space linear isometries.

7.1.1. Gram-Schmidt process. Consider $\mathbf{e}_{1}, \mathbf{e}_{2}, \ldots$ a fixed orthonormal basis system of $\mathcal{H}$. We perform the Gram-Schmidt process as follows:

$$
\begin{aligned}
\mathbf{f}_{\nu 1} & :=T_{\nu}\left(\mathbf{e}_{1}\right) \\
& \vdots \\
\mathbf{f}_{\nu k} & :=T_{\nu}\left(\mathbf{e}_{k}\right)-\sum_{j=1}^{k-1} \frac{\left\langle T_{\nu}\left(\mathbf{e}_{k}\right), \mathbf{f}_{\nu j}\right\rangle}{\left\langle\mathbf{f}_{\nu j}, \mathbf{f}_{\nu j}\right\rangle} \mathbf{f}_{\nu j} \\
& \vdots
\end{aligned}
$$

inductively on $k=1,2, \ldots$.

7.1.2. Uniform upper-bound for $\mathbf{f}_{\nu k}$. By construction, we have that $\left\langle\mathbf{f}_{\nu j}, \mathbf{f}_{\nu k}\right\rangle=0$ whenever $j \neq k$. Consequently,

$$
\left\|T_{\nu}\left(\mathbf{e}_{k}\right)\right\|^{2}=\left\|\mathbf{f}_{\nu k}\right\|^{2}+\sum_{j=1}^{k-1}\left|\left\langle T_{\nu}\left(\mathbf{e}_{k}\right), \mathbf{f}_{\nu j} /\left\|\mathbf{f}_{\nu j}\right\|\right\rangle\right|^{2} .
$$

In particular, we obtain

$$
\left\|\mathbf{f}_{\nu k}\right\| \leq C
$$

and

$$
\sum_{j=1}^{k-1}\left|\left\langle T_{\nu}\left(\mathbf{e}_{k}\right), \mathbf{f}_{\nu j} /\left\|\mathbf{f}_{\nu j}\right\|\right\rangle\right|^{2} \leq C
$$

for all positive integer values of $\nu$ and $k$.

7.1.3. Uniform lower-bound for $\mathbf{f}_{\nu k}$. Note that our construction implies that

$$
\operatorname{Span}\left\{\mathbf{f}_{\nu 1}, \ldots, \mathbf{f}_{\nu k}\right\}=\operatorname{Span}\left\{T_{\nu}\left(\mathbf{e}_{1}\right), \ldots, T_{\nu}\left(\mathbf{e}_{k}\right)\right\}
$$

for every $k=1,2, \ldots$ Hence

$$
\mathbf{f}_{\nu k}=T_{\nu}\left(\mathbf{e}_{k}\right)+\sum_{j=1}^{k-1} \lambda_{j} T_{\nu}\left(\mathbf{e}_{j}\right)
$$


for some $\lambda_{j} \in \mathbb{C}, j=1,2, \ldots$ As a result,

$$
\begin{aligned}
\left\|\mathbf{f}_{\nu k}\right\|^{2} & =\left\|T_{\nu}\left(\mathbf{e}_{k}+\sum_{j=1}^{k-1} \lambda_{j} \mathbf{e}_{j}\right)\right\|^{2} \\
& \geq \frac{1}{C^{2}}\left\|\mathbf{e}_{k}+\sum_{j=1}^{k-1} \lambda_{j} \mathbf{e}_{j}\right\|^{2} \\
& =\frac{1}{C^{2}}\left(1+\sum_{j=1}^{k-1}\left|\lambda_{j}\right|^{2}\right) .
\end{aligned}
$$

In conclusion, we have

$$
\left\|\mathbf{f}_{\nu k}\right\| \geq \frac{1}{C}, \text { for every } \nu, k=1,2, \ldots .
$$

7.2. A sequence of Hilbert space isometries. Consider the mapping $S_{\nu}: \mathcal{H} \rightarrow$ $\mathcal{H}$ defined by

$$
S_{\nu}\left(\sum_{j=1}^{\infty} \alpha_{j} \frac{\mathbf{f}_{\nu k}}{\left\|\mathbf{f}_{\nu k}\right\|}\right)=\sum_{j=1}^{\infty} \alpha_{j} \mathbf{e}_{j}
$$

for every sequence $\left\{\alpha_{j}\right\}_{j=1,2, \ldots}$ of complex numbers satisfying

$$
\sum_{j=1}^{\infty}\left|\alpha_{j}\right|^{2}<\infty
$$

Notice that this defines a Hilbert space isometry for every $\nu=1,2, \ldots$

7.3. The sequence $S_{\nu} \circ T_{\nu}$. Recall the notation

$$
\Sigma_{n}=\operatorname{Span}\left\{\mathbf{e}_{1}, \ldots, \mathbf{e}_{n}\right\}, \text { for } n=1,2, \ldots .
$$

Let us write

$$
A_{\nu} \equiv S_{\nu} \circ T_{\nu}
$$

for each $\nu$. Then, as its construction shows, the sequence $A_{\nu}$ satisfies:

- $A_{\nu}$ maps $\Sigma_{n}$ onto itself as a $\mathbb{C}$-linear isomorphism.

- Both $\left\|\left.A_{\nu}\right|_{\Sigma_{n}}\right\|$ and $\left\|\left.A^{-1}\right|_{\Sigma_{n}}\right\|$ are bounded by the positive constant $C$, independent of $\nu$ and $n$.

Therefore we may choose a subsequence of $A_{\nu}$ which converges in norm to an operator

$$
A: \bigcup_{n=1}^{\infty} \Sigma_{n} \rightarrow \bigcup_{n=1}^{\infty} \Sigma_{n}
$$

which is invertible on each $\Sigma_{n}$ for $n=1,2, \ldots$

We now extend $A$ to an operator on all of $\mathcal{H}$. Let $\epsilon>0$ be given. For each $\mathbf{v} \in \mathcal{H}$ with $\|\mathbf{v}\|=1$, we choose a positive integer $k$ such that

$$
\left\|\mathbf{v}-\pi_{k}(\mathbf{v})\right\|<\frac{\epsilon}{3 C} .
$$


Here $\pi_{k}: \mathcal{H} \rightarrow \Sigma_{n}$ denotes the orthogonal projection, as before. Notice, in particular, that $\left\|\pi_{k}(\mathbf{v})\right\| \leq\|\mathbf{v}\| \leq 1$. Then, for this $k$, we may choose $N>0$ such that

$$
\left\|A_{\nu}\left(\pi_{k}(\mathbf{v})\right)-A_{\mu}\left(\pi_{k}(\mathbf{v})\right)\right\| \leq \frac{\epsilon}{3}, \text { for all } \nu, \mu>N .
$$

Then we obtain, for every $\nu, \mu>N$, that

$$
\begin{aligned}
\left\|A_{\nu}(\mathbf{v})-A_{\mu}(\mathbf{v})\right\| \leq & \left\|A_{\nu}\left(\mathbf{v}-\pi_{k}(\mathbf{v})\right)\right\|+\left\|A_{\nu}\left(\pi_{k}(\mathbf{v})\right)-A_{\mu}\left(\pi_{k}(\mathbf{v})\right)\right\| \\
& \quad+\left\|A_{\mu}\left(\mathbf{v}-\pi_{k}(\mathbf{v})\right)\right\| \\
& <\epsilon .
\end{aligned}
$$

The completeness of $\mathcal{H}$ shows that

$$
\widehat{A}(\mathbf{v}) \equiv \lim _{\nu \rightarrow \infty} A_{\nu}(\mathbf{v})
$$

exists for every $\mathbf{v} \in \mathcal{H}$. It is obvious that $\widehat{A}$ is linear and is the unique extension of $A$. Now we show that $\widehat{A}$ is bounded. To see this, let $\mathbf{v} \in \mathcal{H}$ satisfy $\|\mathbf{v}\|=1$. Then choose $\nu$ such that $\left\|\widehat{A}(\mathbf{v})-A_{\nu}(\mathbf{v})\right\| \leq 1$. Thus

$$
\begin{aligned}
\|\widehat{A}(\mathbf{v})\| & \leq\left\|\widehat{A}(\mathbf{v})-A_{\nu}(\mathbf{v})\right\|+\left\|A_{\nu}(\mathbf{v})\right\| \\
& \leq 1+C .
\end{aligned}
$$

Repeating the same process with $A_{\nu}^{-1}$, and choosing subsequences whenever necessary, we arrive at the following conclusion:

Proposition 7.1. Every subsequence of $A_{\nu}$ above has itself a subsequence that converges to a bounded operator $\widehat{A}: \mathcal{H} \rightarrow \mathcal{H}$, uniformly on each finite slice $\Sigma_{n}=$ $\operatorname{Span}\left\{\mathbf{e}_{1}, \ldots, \mathbf{e}_{n}\right\}, n=1,2, \ldots$ Moreover, $\widehat{A}$ is invertible and preserves $\Sigma_{n}$ for each $n$.

Rephrasing, we have

Proposition 7.2. Let $T_{\nu}: \mathcal{H} \rightarrow \mathcal{H}$ be invertible $\mathbb{C}$-linear operators with a constant $C>0$ such that

$$
\left\|T_{\nu}\right\| \leq C \quad \text { and } \quad\left\|T_{\nu}^{-1}\right\| \leq C
$$

for every $\nu=1,2, \ldots$ Then there exists a sequence $S_{\nu}: \mathcal{H} \rightarrow \mathcal{H}$ of Hilbert space isometries such that the sequence $A_{\nu} \equiv S_{\nu} \circ T_{\nu}$ satisfies the following:

(i) Each $S_{\nu} \circ T_{\nu}$ maps $\Sigma_{n}$ isomorphically onto $\Sigma_{n}$.

(ii) There exists a subsequence $A_{\nu_{j}}$ such that both $A_{\nu_{j}}$ and $A_{\nu_{j}}^{-1}$ converge to invertible bounded operators, respectively, each of which maps $\Sigma_{n}$ isomorphically onto itself for every $n=1,2, \ldots$.

7.4. The second adjustment of the scaling sequence. We now apply the above arguments to the scaling sequence. We begin with the following observation on the derivatives of the scaling mappings.

7.4.1. The sequence $d \omega_{\nu}(\mathbf{q} ; \cdot)$. We write $T_{\nu}(\cdot)=d \omega_{\nu}(\mathbf{q} ; \cdot)$ for each $\nu$. Then we prove

Proposition 7.3. There exists a subsequence (which we also denote by $T_{\nu}$ ) of $T_{\nu}$ which admits a constant $C>0$ such that

$$
\frac{\|\mathbf{v}\|}{C} \leq\left\|T_{\nu}(\mathbf{v})\right\| \leq C\|\mathbf{v}\|
$$


and

$$
\frac{\|\mathbf{v}\|}{C} \leq\left\|T_{\nu}^{-1}(\mathbf{v})\right\| \leq C\|\mathbf{v}\|
$$

for every $\mathbf{v} \in \mathcal{H}$.

Proof. Recall that, choosing a subsequence, we may arrange that:

(1) there exists a constant $\delta>0$ independent of $\nu$ such that $\mathbb{B}(\mathbf{q} ; \delta) \subset \Omega_{\nu}$ for every $\nu$;

(2) there exists a constant $r>1$ independent of $\nu$ such that $\omega_{\nu}\left(\Omega_{\nu}\right) \subset \mathbb{B}(\mathbf{0} ; r)$;

(3) $\omega_{\nu}(\mathbf{q})=\mathbf{0}$.

We use the notation $k_{G}: G \times \mathcal{H} \rightarrow \mathbb{R}$ to denote the (infinitesimal) Kobayashi metric of the domain $G \subset \mathcal{H}$. (Notice that the notion of Kobayashi metric admits a natural generalization from the finite-dimensional case, at least for our Hilbert space $\mathcal{H}$.) We immediately obtain

$$
\begin{aligned}
\frac{1}{r}\left\|T_{\nu}(\mathbf{v})\right\|_{\mathcal{H}} & =k_{\mathbb{B}(\mathbf{0} ; r)}\left(\mathbf{0} ; T_{\nu}(\mathbf{v})\right) \\
& =k_{\mathbb{B}(\mathbf{0} ; r)}\left(\omega_{\nu}(\mathbf{q}) ; d \omega_{\nu}(\mathbf{q} ; \mathbf{v})\right) \\
& \leq k_{\Omega_{\nu}}(\mathbf{q} ; \mathbf{v}) \\
& \leq k_{\mathbb{B}(\mathbf{q} ; \delta)}(\mathbf{q} ; \mathbf{v}) \\
& =\frac{1}{\delta}\|\mathbf{v}\| .
\end{aligned}
$$

This yields that

$$
\left\|T_{\nu}\right\| \leq \frac{r}{\delta}, \text { for all } \nu=1,2, \ldots .
$$

A similar argument on $T_{\nu}^{-1}$ yields a uniform bound for $\left\|T_{\nu}^{-1}\right\|$. Altogether, we arrive at the desired conclusion.

7.4.2. Adjusted scaling sequence $\alpha_{\nu}$. Notice first that, for any subsequence of a scaling sequence $\omega_{\nu}$, we may now choose a sequence $S_{\nu}$ of $\mathbb{C}$-linear isometries such that a subsequence (again we abuse notation and let $\alpha_{\nu}$ also be the subsequence)

$$
\alpha_{\nu} \equiv S_{\nu} \circ \omega_{\nu}: \Omega_{\nu} \rightarrow \mathcal{H}
$$

satisfies the following conditions:

(i) For every $\nu=1,2, \ldots$, the operator

$$
A_{\nu} \equiv d \alpha_{\nu}(\mathbf{q} ; \cdot): \mathcal{H} \rightarrow \mathcal{H}
$$

and its inverse $A_{\nu}^{-1}$ have their norms bounded by a constant independent of $\nu$.

(ii) Each $A_{\nu}$ maps $\Sigma_{n}=\operatorname{Span}\left\{\mathbf{e}_{1}, \ldots, \mathbf{e}_{n}\right\}$ isomorphically onto itself, for every $n=1,2, \ldots$.

(iii) The sequence $A_{\nu}$ converges to a bounded invertible operator (with a bounded inverse), say $\widehat{A}$, on each $\Sigma_{n}$ for $n=1,2, \ldots$.

Notice that, for every $r>1$, there exists $N>0$ such that $\omega_{\nu}\left(\Omega_{\nu}\right) \subset \mathbb{B}(\mathbf{0} ; r)$ for every $\nu>N$. Since the $S_{\nu}$ are isometries, we obviously have the same property for the newly adjusted scaling sequence $\alpha_{\nu}$. Hence we may choose a weak limit in the sense of Section 5, which eventually yields the limit holomorphic mapping $\widehat{\alpha}: \Omega \rightarrow \mathbb{B}$. 
Notice that

$$
d \widehat{\alpha}(\mathbf{q} ; \cdot)=\widehat{A} .
$$

Choosing a subsequence once again if necessary, we see that there exists a weak limit mapping $\beta: \mathbb{B} \rightarrow \mathcal{H}$ of the sequence

$$
\alpha_{\nu}^{-1}: \mathbb{B}(\mathbf{0} ; s) \rightarrow \Omega
$$

for $s<1$. The preceding arguments then show that:

(i) $\left[d \alpha_{\nu}^{-1}\right](\mathbf{0} ; \cdot)$ maps each $\Sigma_{n}$ isomorphically onto itself, and as a result it converges to $\widehat{A}^{-1}$ on each finite-dimensional slice $\Sigma_{n}$. Consequently, we have

$$
d \beta(\mathbf{0} ; \cdot)=\widehat{A}^{-1} .
$$

(ii) The limit mapping $\widehat{\beta}$ is well-defined on the unit ball $\mathbb{B}$ and satisfies $\widehat{\beta}(\mathbb{B}) \subset \widehat{\Omega}$, where $\widehat{\Omega}$ denotes the (open) convex hull of $\Omega$.

(In general, $\widehat{\beta}(\mathbb{B})$ is contained in the closure of $\widehat{\Omega}$. But, since $\widehat{\beta}(\mathbf{0})=\mathbf{q}$, the Maximum Principle implies the conclusion above.)

Finally, we arrive at

7.5. Proof of the Main Theorem. We are now ready to give the proof of the Main Theorem (Theorem 3.1), which is

Main Theorem. Every bounded convex domain $\Omega$ in a separable Hilbert space $\mathcal{H}$ admitting a $C^{2}$ strongly pseudoconvex boundary point at which an automorphism orbit accumulates is biholomorphic to the open unit ball of $\mathcal{H}$.

Proof. The scaling arguments above yield the holomorphic mappings

$$
\widehat{\alpha}: \Omega \rightarrow \mathbb{B} \text { and } \widehat{\beta}: \mathbb{B} \rightarrow \Omega=\widehat{\Omega}
$$

satisfying the conditions:

(1) $\widehat{\alpha}(\mathbf{q})=\mathbf{0}$,

(2) $\widehat{\beta}(\mathbf{0})=\mathbf{q}$,

(3) $d(\widehat{\alpha} \circ \widehat{\beta})(\mathbf{0} ; \mathbf{v})=\mathbf{v}$ and $d(\widehat{\beta} \circ \widehat{\alpha})(\mathbf{q} ; \mathbf{v})=\mathbf{v}$ for every $\mathbf{v} \in \mathcal{H}$.

Now we apply the infinite-dimensional version of Cartan's Uniqueness Theorem (essentially identical with the original finite-dimensional version; see $[\mathrm{BM}]$ ). Then both $\widehat{\alpha} \circ \widehat{\beta}$ and $\widehat{\beta} \circ \widehat{\alpha}$ are equal to the identity map. Therefore $\alpha: \Omega \rightarrow \mathbb{B}$ is in particular a biholomorphic mapping. This completes the proof.

\section{Concluding Remarks}

The main result of this paper - a version of the Wong/Rosay theorem in a separable Hilbert space - has only been proved here for convex domains. The role of convexity in several complex variables has been long established (see [LEM1, [KRA2]). Nevertheless, it is not well understood. In particular, there is a rather poor understanding of convexity from the biholomorphically invariant point of view. We have no examples to indicate whether our result ought to be true for more general classes of domains.

Of course the exploration of domains of finite type will be an entirely new world. The situation for such domains in dimension 2 is now fairly well in hand. In higher dimensions there is yet much to be understood, and we are still some distance from having any true grasp of the infinite-dimensional situation. 


\section{REFERENCES}

[BP1] Bedford, E. and Pinchuk, S., Domains in $\mathbb{C}^{2}$ with non-compact holomorphic automorphism group (translated from Russian), Math. USSR-Sb. 63(1989), 141-151.

[BP2] Bedford, E. and Pinchuk, S., Domains in $\mathbb{C}^{n+1}$ with non-compact automorphism groups, J. Geom. Anal. 1(1991), 165-191. MR 92f:32024

[BP3] Bedford, E. and Pinchuk, S., Convex domains with non-compact automorphism group (translated from Russian), Russian Acad. Sci. Sb. Math. 82(1995), 1-20.

[BP4] Bedford, E. and Pinchuk, S., Domains in $\mathbb{C}^{2}$ with non-compact automorphism group, Indiana Univ. Math. J. 47(1998), 199-222. MR 99e:32058

[Ber1] Berteloot, F., Sur certains domaines faiblement pseudoconvexes dont le groupe d'automorphismes analytiques est non compact, Bull. Sci. Math. (2) 114(1990), 411420. MR 91j:32035

[Ber2] Berteloot, F., Un principe de localisation pour les domaines faiblement pseudoconvexes de $\mathbb{C}^{2}$ dont le groupe d'automorphismes holomorphes est non compact, Colloque d'Analyse Complexe et Géométrie (Marseille, 1992), Asterisque 217(1993), 13-27. MR 94k:32019

[Ber3] Berteloot, F., Characterization of models in $\mathbb{C}^{2}$ by their automorphism groups, Internat. J. Math. 5(1994), 619-634. MR 95i:32040

[BeCo] Berteloot, F. and Cœuré G., Domaines de $\mathbb{C}^{2}$, pseudoconvex et de type fini ayant un groupe non compact d'automorphismes, Ann. Inst. Fourier Grenoble 41(1991), 77-88. $\operatorname{MR}$ 92j:32053

[BM] Bochner, S. and Martin, W., Several complex variables, Princeton Univ. Press, 1948. MR 10:366a

[FIK1] Fu, S., Isaev, A. V. and Krantz, S. G., Examples of domains with non-compact automorphism groups, Math. Res. Letters 3(1996), 609-617. MR 97f:32047

[FIK2] Fu, S., Isaev, A. V. and Krantz, S. G., Reinhardt domains with non-compact automorphism groups, Math. Res. Letters 3(1996), 109-122. MR 97c:32043

[Fr] Frankel, S., Complex geometry of convex domains that cover varieties, Acta Math. 163(1989), 109-149. MR 90i:32037.

[GK1] R. E. Greene and S. G. Krantz, Characterizations of certain weakly pseudo-convex domains with non-compact automorphism groups, in Complex Analysis Seminar, Springer Lecture Notes Vol. 1268 (1987), 121-157. MR 89c:32044]

[IK1] A. Isaev and S. G. Krantz, Domains with non-compact automorphism group: A survey, Advances in Math. 146(1999), 1-38. MR 2000i:32053

[IK2] Isaev, A. V. and Krantz, S. G., On the boundary orbit accumulation set for a domain with non-compact automorphism group, Michigan Math. J. 43(1996), 611-617. MR 97m:32048

[IK3] Isaev, A. V. and Krantz, S. G., Finitely smooth Reinhardt domains with non-compact automorphism group, Illinois. J. Math. 41(1997), 412-420. MR 98i:32054

[IK4] Isaev, A. V. and Krantz, S. G., Hyperbolic Reinhardt domains with non-compact automorphism group, Pacific J. Math. 184(1998), 149-160. MR 99h:32003

[Ki1] Kim, K.-T., Domains with non-compact automorphism groups, Recent Developments in Geometry (Los Angeles, CA, 1987), 249-262, Contemp. Math. 101, Amer. Math. Soc.,1989. MR 90m:32036

[Ki2] Kim, K.-T., Complete localization of domains with non-compact automorphism groups, Trans. Amer. Math. Soc. 319(1990), 139-153. MR 90i:32035

[Ki3] Kim, K.-T., Domains in $\mathbb{C}^{n}$ with a piecewise Levi flat boundary which possess a noncompact automorphism group, Math. Ann. 292(1992), 575-586. MR 93h:32024

[Ki4] Kim, K.-T., Geometry of bounded domains and the scaling techniques in several complex variables, Lecture Notes Series 13, Seoul National University, Research Institute of Mathematics, Global Analysis Research Center, Seoul, 1993. MR 96c:32036

[Ki5] Kim, K.-T., On a boundary point repelling automorphism orbits, J. Math. Anal. Appl. 179(1993), 463-482. MR 94m:32048

[Ki6] Kim, K.-T., Two examples for scaling methods in several complex variables, RIMGARC Preprint Series, Seoul National University 95-53(1995).

[KIKR] Kim, K.-T. and Krantz, S. G., A crash course in the function theory of several complex variables, Complex geometric analysis in Pohang(1997), 3-37, Contemp. Math. 222, American Math. Society, Providence, RI, 1999. MR 99g:32002 
[KRA1] Krantz, S. G., Function Theory of Several Complex Variables, $2^{\text {nd }}$ Ed., Wadsworth, Belmont, 1992. MR 93c:32001

[KRA2] Krantz, S. G., Convexity in complex analysis, Proc. Symp. Pure Math., vol. 52 (E. Bedford, J. D'Angelo, R. Greene, and S. Krantz eds.), American Mathematical Society, Providence, R.I., 1991. MR 92g:32001

[LEM1] Lempert, L., La metrique de Kobayashi et la representation des domains sur la boule, Bull. Soc. Math. France 109(1981), 427-474. MR 84d:32036

[LEM2] Lempert, L., The Dolbeault complex in infinite dimensions, J. AMS 11(1998), 485-520. MR 99f:58007

[Mu Mujica, J., Complex analysis in Banach spaces, North-Holland, 1986. MR 88d:46084

[NAR] Narasimhan, R., Several Complex Variables, University of Chicago Press, Chicago, 1971. MR 49:7470

[ROS] Rosay, J.-P., Sur une characterization de la boule parmi les domains de $\mathbb{C}^{n}$ par son groupe d'automorphismes, Ann. Inst. Four. Grenoble XXIX(1979), 91-97.

[WON] Wong, B., Characterization of the ball in $\mathbb{C}^{n}$ by its automorphism group, Invent. Math. 41(1977), 253-257.

Department of Mathematics, Pohang University of Science and Technology, Pohang 790-784, The Republic of Korea

E-mail address: kimkt@postech.edu

Department of Mathematics, Washington University in St. Louis, St. Louis, Missouri 63130

E-mail address: sk@math.wustl.edu 